\title{
The Contribution of Cement Companies to the Achievement of Sustainable Development Goals and its Effect in Building Trust in their Communities
}

Adriana Martínez Lecuona, Candidata PhD (UPAEP)

Profesor Investigador de Tiempo Completo,

Universidad Autónoma del Estado de Hidalgo (UAEH), México

Manuel González Pérez, PhD

Profesor Investigador de Tiempo Completo, Universidad Popular Autónoma del Estado de Puebla (UPAEP),

Miembro del Sistema Nacional de Investigación Nivel 1, México

Paola Selene Vera Martínez, PhD

Profesor Investigador de Tiempo Completo, Universidad Nacional Autónoma de México (UNAM), México

Genoveva Rosano Romero, PhD

Profesor Investigador de Tiempo Completo,

Universidad Popular Autónoma del Estado de Puebla (UPAEP), México

Martín Badillo Maldonado, Estudiante PhD (UPAEP)

Doi: 10.19044/esj.2017.v13n29p358 URL:http://dx.doi.org/10.19044/esj.2017.v13n29p358

\begin{abstract}
Companies have reoriented their actions in contributing towards the attainment of the Sustainable Development Goals (SDGs). The SDGs enhances the reputation of enterprises and builds confidence in their stakeholders. People's confidence toward the company generates favorable attitudes and behaviors. Therefore, the purpose of this paper is to identify the level of contribution of cement companies to achieve Sustainable Development Goals and its effect on the trust of its local communities. In achieving the purpose of this work, we study the perception of people about the actions that the company makes in favor of sustainable development. The study variables correspond to 9 of 17 SDGs classified into two categories (people and environment). The nine variables are compared with confidence. Data are also analyzed with statistical standardization and chi-square tools. The results show that only $40 \%$ of the actions carried out by the company contribute to the achievement of the SDGs. However, there is no significant relationship between the population's confidence in the company and the actions that the firm makes in favor of sustainable development.
\end{abstract}


Keywords: Sustainability, Sustainable Development Objectives, Trust, Reputation

\section{Resumen}

Las empresas reorientan sus acciones para contribuir al logro de los Objetivos de Desarrollo Sostenible (ODS). Los ODS mejoran la reputación de las empresas y fomentan la confianza en sus grupos de interés. La confianza de la gente hacia la empresa genera actitudes y comportamientos favorables. Por lo tanto, el propósito de este trabajo es identificar el nivel de contribución de una empresa de cemento para lograr los ODS y su efecto en la confianza de sus comunidades locales. Para alcanzar el propósito de este trabajo, estudiamos la percepción de las personas sobre las acciones que la empresa hace a favor del desarrollo sostenible. Las variables del estudio corresponden a 9 de 17 ODS clasificadas en dos categorías (personas y medio ambiente). Las nueve variables se comparan con la confianza. Los datos también se analizan con estandarización estadística y herramientas de chi-cuadrado. Los resultados muestran que sólo el 40\% de las acciones realizadas por la empresa contribuyen a la consecución de los ODS. Sin embargo, no hay relación entre la confianza de la población en la empresa y las acciones que la empresa hace a favor del desarrollo sostenible.

Palabras clave: Sostenibilidad, Objetivos de Desarrollo Sostenible, Confianza, Reputación

\section{Introducción}

En septiembre de 2015, la Asamblea General de las Naciones Unidas aprobó los ODS en el documento titulado "Transformar nuestro mundo: la Agenda 2030 para el Desarrollo Sostenible”. En su contenido, el documento se define como "un plan de acción en favor de las personas, el planeta y la prosperidad" (Naciones Unidas, 2015, p.1).

Este plan de acción presenta 17 objetivos que constituyen el soporte a través del cual los países podrán diseñar sus planes y encaminar esfuerzos para poner fin a la pobreza, reducir la desigualdad y disminuir el impacto al medio ambiente.

Los ODS no son jurídicamente obligatorios para ningún sector; sin embargo, se espera que a través de una Alianza Mundial para el Desarrollo Sostenible, participen los gobiernos, la sociedad civil, el sistema de las Naciones Unidas y otras instancias.

Con relación al sector privado, algunos organismos internacionales han aportado numerosas herramientas y plataformas para facilitar a las empresas la tarea de comprender e integrar los ODS como son: SDG 
Compass (Brújula de los ODS), Business \& Sustainable Development Commission, Action 2020, SDG Business Hub, entre otras.

El SDG Compass es una guía para las empresas que explica cómo interpretar y adoptar los ODS en su estrategia de negocio. Para incentivar la participación en la adopción de los ODS, el SDG Compass señala algunos beneficios que pueden obtener las empresas, como son: identificar futuras oportunidades de negocio, mejorar el valor de la sostenibilidad empresarial, estabilizar sociedades y mercados, usar un lenguaje común y un propósito compartido, así como fortalecer las relaciones con las partes interesadas y estar al día con el desarrollo de políticas (PMNU, WBCSD \& GRI, 2015).

Particularmente, con relación a fortalecer las relaciones con las partes interesadas, el SDG Compass destaca que "las empresas que alinean sus prioridades con los ODS, pueden fortalecer su compromiso con los clientes, empleados y con otras partes interesadas; aquellas que no lo hagan, estarán expuestas a crecientes riesgos legales y de reputación" (PMNU, WBCSD \& GRI, 2015, p.4).

En el mismo sentido, Remacha (2017) afirma que "integrar los ODS será una manera de lograr una actitud positiva de los grupos de interés hacia la entidad, obtener su confianza y mantener la legitimidad para operar" (Remacha, 2017, p. 8).

Sin embargo, han existido algunos estudios que muestran cómo las empresas a menudo son acusadas de "adornar" sus actividades sociales provocando un impacto negativo en su reputación (Bouten, Everaert, Liedekerke, \& De Moor, 2011; Font, Walmsley, Cogotti, McCombes, \& Hdusler, 2012).

Por su parte, Edelman $(2014,2015)$ realizó una encuesta a 500 personas en Estados Unidos y China así como a 200 personas en otros 25 países, con el propósito de mostrar el Índice de Confianza, en las instituciones de gobierno, empresa, medios de comunicación y ONG. Entre los años de 2014 y 2015 , los resultados de las encuestas muestran que más del $50 \%$ de los países participantes presentan un descenso global de la confianza hacia las empresas del $59 \%$ al $57 \%$. Las principales razones por las que algunos encuestados manifiestan menor confianza en las empresas son: por la corrupción o fraude, por incompetencia en la gestión de sus organizaciones y por problemas de transparencia en su información (Edelman, 2014, 2015).

En el caso particular de México, la tendencia de la confianza hacia las empresas, durante los años de 2008 a 2015 presenta una confianza promedio del $76 \%$. Sin embargo, la tendencia muestra una disminución del $10 \%$ en los últimos tres años, (82\% en $2013,73 \%$ en 2014 y $72 \%$ en 2015 ) (Edelman, 2013, 2014, 2015).

La disminución de la confianza hacia las empresas que realizan acciones en favor de la sustentabilidad en México, de acuerdo a diversos 
autores, se debe a que el trato e imagen que las empresas muestran ante el cliente es "amable" con el objetivo de vender más (Barroso, 2008). De la misma forma, porque las empresas son esencialmente asistencialistas, paternalistas y caritativas con sus comunidades sin atender a las necesidades "reales" y porque sus actividades se encuentran opuestas a la realidad de otras partes con quienes se relacionan (Saldaña, 2010). Asimismo, se debe a que las "buenas prácticas" corporativas se dedican solo a vender valores y no productos (García, 2012). Finalmente, porque a pesar de que los empresarios poseen experiencia y liderazgo para gestionar sus organizaciones, ello no implica verdaderas "prácticas responsables” (Montañez \& Gutiérrez, 2014).

Hoy en día, personas de todo el mundo tienen mayor entendimiento y consciencia sobre la importancia en que las empresas realicen acciones en favor de la sustentabilidad. La consultoría PwC (2015), con el objetivo de saber el nivel de consciencia de los ciudadanos sobre los ODS, realizaron una encuesta a 2015 personas en diferentes países. Entre los encuestados, el 90\% manifestó que es importante que las empresas se adhieran a los ODS; afirmando que el $78 \%$ de los mismos, son más propensos a comprar los bienes y servicios de las empresas que se habían adherido a los objetivos.

Por tanto, es de gran importancia para las empresas elegir una estrategia solvente que permita incrementar los niveles de confianza de la sociedad hacia éstas, debido a que la credibilidad genera un esquema de incentivos o sanciones; económicos, sociales y políticos, que favorecen la generación de riqueza con perspectiva sustentable, mismos que permiten armonizar los objetivos financieros de la empresa con el interés público de las comunidades en las que opera (IPADE \& GESOC, 2011).

A partir del $1^{\circ}$ de enero de 2016, fecha en que entraron en vigor los ODS, numerosas empresas en todo el mundo iniciaron la adhesión a estos objetivos. En este sentido, la consultoría PwC (2015), a partir de una encuesta a 986 compañías miembros del Pacto Mundial de Naciones Unidas (UNGC) y miembros de Global Reporting Initiative (GRI), señala que el $71 \%$ de éstas ya se habían planteado cómo comprometerse a los ODS y; el $41 \%$, afirmaba que los integrarían a su estrategia durante los próximos cinco años.

En el mismo sentido, Garcidueñas (2015) señaló a 20 empresas que ya trabajaban para sumarse al logro de los ODS, entre ellas Cementos Mexicanos (Cemex). Esta empresa, desde años atrás, ya contaba con su programa Patrimonio Hoy, una iniciativa de negocios inclusivos donde pretende contribuir al desarrollo social de las comunidades, brindándoles una vivienda digna a través de la autoconstrucción y de créditos de fácil acceso. Otras iniciativas que también ya contribuían al logro de los ODS son la instalación de ecotecnias en poblaciones vulnerables para el aprovechamiento de los recursos, así como programas para fortalecer a las 
mujeres, a través de centros de desarrollo comunitarios en los lugares donde la empresa realiza sus operaciones. Con estas acciones, Garcidueñas afirmaba que Cemex ya daba cumplimiento con los objetivos: 5, 7, 10, 11, 15.

Por su parte, la empresa Cemex informa, a través de su reporte de sustentabilidad (Cemex, 2016), haber adherido a su estrategia de negocio los ODS así como las acciones que realizó durante el año 2015 que contribuyen al logro de estos objetivos.

Ante este planteamiento, se presentan los siguientes cuestionamientos: ¿Cuál es la percepción de los habitantes de las comunidades locales sobre las acciones que realiza la empresa Cemex para contribuir al logro de los ODS? y ¿Estas acciones generan confianza en las personas hacia la empresa?

Para responder a estas interrogantes, el propósito del trabajo es identificar el nivel de contribución al logro de los ODS de la empresa Cemex, Planta Atotonilco; a través de las acciones que realiza en favor de la sustentabilidad en sus comunidades locales; así como la relación de estas acciones con la confianza de los pobladores hacia la empresa.

El resultado esperado al término de la investigación se plantea en dos direcciones: Por una parte, podemos afirmar que el nivel de contribución de la empresa Cemex al logro de los ODS, a través de las acciones en favor de las personas, es significativa; y que las acciones en favor al medio ambiente no lo son.

Por otra parte, podemos aseverar que la confianza de los habitantes de sus comunidades locales depende de las acciones de sustentabilidad que realiza la empresa para contribuir al logro de los ODS.

\section{Revisión de la Literatura}

El Informe Brundtland, hace operativo el concepto de sustentabilidad al hablar del desarrollo sostenible el cual define como "aquel desarrollo que satisface las necesidades del presente sin comprometer la capacidad de las generaciones futuras para satisfacer sus propias necesidades". Esta definición comprende dos enfoques: uno social, relativo al hombre para satisfacer "las necesidades esenciales de los pobres del mundo" y uno ecológico, al hablar de "la capacidad del medio ambiente para satisfacer las necesidades presentes y futuras" (Naciones Unidas, 1987, p. 37). En este sentido, el desarrollo sostenible puede dividirse conceptualmente en tres partes: económico, ambiental y social. Se consideran las tres dimensiones por la relación entre el bienestar social, el equilibrio con el medio ambiente y la bonanza económica (Barrios, 2010).

En este sentido, en septiembre de 2015, los jefes de estado y de gobierno; así como los altos representantes de las diferentes naciones del 
mundo, reunidos en la sede de las Naciones Unidas en Nueva York, han establecido una nueva ruta a seguir para los próximos 15 años: los 17 Objetivos de Desarrollo Sostenible para favorecer el desarrollo sostenible (Naciones Unidas, 2015).

Los ODS, se traducen en 169 metas que abarcan aspectos sociales, ambientales y económicos. Estos objetivos buscan generar un impacto positivo en los siguientes atributos: 1) Fin de la pobreza, 2) Hambre cero, 3) Salud y bienestar, 4) Educación inclusiva, equitativa y de calidad, 5) Igualdad de género, 6) Agua limpia y saneamiento, 7) Energía asequible y no contaminante, 8) Trabajo decente y crecimiento económico, sostenido, inclusivo y sostenible, 9) Infraestructuras resilientes, promover la industrialización inclusiva y sostenible, 10) Reducción de las desigualdades, 11) Ciudades y comunidades sostenibles, 12) Producción y consumo responsables, 13) Acciones ante el cambio climático, 14) Vida submarina, 15) Vida de ecosistemas terrestres, 16) Paz, justicia e instituciones solidarias, y 17) Alianzas para lograr los objetivos (Naciones Unidas, 2015).

La implementación de los ODS inicia el $1^{\circ}$ de enero de 2016, contemplando la mayoría de las metas para 2030 y algunas más urgentes para 2020. Su alcance se extiende a todos los países, independientemente de cual sea su grado de desarrollo y cada nación será responsable de establecer sus prioridades con base en la situación en la que se encuentre.

La responsabilidad de desarrollar políticas, planes y programas de desarrollo sostenible y de llevar un seguimiento y un control de sus avances para lograr los ODS son los países. Asimismo, los países tienen la responsabilidad de promover la cooperación entre los diferentes actores y buscan movilizar los esfuerzos a nivel global en torno a un conjunto de metas comunes. Por este motivo, los ODS reconocen explícitamente el rol fundamental que las empresas pueden y deben desempeñar en su logro (Remacha, 2017).

La contribución de las empresas a los ODS puede abordarse desde tres enfoques: 1) la implementación de iniciativas con relación a las operaciones de la empresa para reducir y eliminar los impactos negativos y potenciar aquellos que sean positivos para los grupos de interés, 2) el desarrollo de productos y servicios innovadores, para generar nuevas oportunidades de negocio, y 3) el desarrollo de acciones filantrópicas mediante las que se busca mejorar las condiciones sociales y ambientales de los entornos en los que opera o no la compañía, y que tienen principalmente un beneficio reputacional (Naciones Unidas, 2015).

Sin embargo, la confianza constituye un factor determinante para sumarse a la participación de las personas en cualquier propósito en el que sea involucrada a colaborar. En este sentido, la confianza puede definirse como el grado de fe, credibilidad y hasta complicidad que puede existir entre 
dos o más individuos. Esta afirmación puede explicarse porque quien confía es consciente de las capacidades, actitudes y aptitudes de la persona en quien deposita su confianza, por esta razón dirige estos elementos de la mejor forma para conseguir un objetivo común (Rojas \& Marin, 2006).

De la misma forma, la confianza es percibida como la esperanza firme que se tiene de alguien o algo; es clave para el capital relacional en las organizaciones y los resultados que se obtienen, una vez alcanzada, son la lealtad y el compromiso (Hewett \& Bearden, 2001).

Por su parte, los profesionales de la estrategia sugieren que la confianza es una fuente que tiene el potencial de proveer una fuerte y sostenible ventaja competitiva (Barney \& Hansen, 1995). Sin embargo, las bases para la construcción de la confianza están en adquirir compromisos sobre los temas y asuntos que son relevantes para los ciudadanos (como respuesta a lo que esperan de la organización los grupos de interés) y después cumplirlos. Por lo tanto, es un requisito fundamental que aquello que comunique una organización después lo cumpla en la realidad; esto es aplicable a una persona, a un profesional, a una empresa, institución o país (Bourdieu, 2000).

Es así como las empresas al obtener confianza elevan su reputación, entendida esta última como el conjunto de evaluaciones colectivas producidas por la corporación en los grupos de interés motivando conductas capaces de generar valor a la organización (Corporate Excellence, 2012). En consecuencia, una reputación mal gestionada puede poner en peligro la continuidad empresarial (Fombrun \& Van Riel, 2004). La clave para construir empresas, instituciones, ciudades o países con buena reputación es hacer lo que se ha dicho que se va a hacer, hacerlo bien y comunicarlo.

La buena reputación impacta en las actitudes y comportamientos favorables de los grupos de interés que son clave para la supervivencia y el crecimiento de una empresa. La buena reputación incrementa la atracción de los consumidores, del capital, del talento, facilita la internacionalización de las empresas y permite alinear los intereses y voluntades de toda la organización para conseguir los objetivos de negocio (Quevedo, 2003).

La confianza en las Instituciones o empresas está basada en los valores compartidos, que se definen como las creencias, preferencias, metas y criterios de elección de metas que se desarrollan y transmiten a través de la cultura, la sociedad y la personalidad dando lugar a un determinado comportamiento (Fields, 2002).

Por su parte, Lewicki, McAllister y Bies (1998), afirman que para generar confianza en necesario poseer intereses, metas, objetivos y valores comunes.

Asimismo, Jones y George (1998) argumentan que compartir valores es la mejor forma para generar confianza. 
Si se espera la contribución de todos los sectores de la sociedad para el logro de los ODS, cada individuo y sector de la sociedad en todo el mundo, debe ser congruente entre lo que dice, piensa y hace; deben compartir valores para generar confianza.

\section{Metodología}

El dominio del estudio se ubica en Industria del cemento por ser considerado uno de los sectores de mayor impacto social y ambiental (Martínez, 2014). Particularmente, la investigación se desarrolla en el análisis del informe de sustentabilidad de la empresa Cemex (2016) y en el estudio de la percepción de las comunidades locales de la empresa en su Planta Atotonilco. Se eligió la empresa Cemex por ocupar el tercer lugar como líder en la industria del cemento a nivel global, contando con $87,1 \mathrm{Mt} /$ año de capacidad de producción de cemento a través de 51 plantas integradas y 9 plantas de molienda (Edwards, 2016). En el mercado nacional, la empresa se posiciona como líder con una participación del $52 \%$ a través de 15 plantas localizadas en 10 estados del país (CANACEM, 2017). A nivel local, la Planta ubicada en el centro del municipio Atotonilco de Tula, en el estado de Hidalgo; es una de las más grandes y con mayor antigüedad.

De acuerdo al propósito planteado, las hipótesis de investigación son:

1. Las acciones de sustentabilidad que realiza Cemex dirigidas al beneficio de las personas sí contribuyen al logro de los ODS y las acciones dirigidas a la conservación del medio ambiente no.

2. La confianza de las personas de la población hacia la empresa, depende de las acciones que ésta realiza para contribuir al logro de los ODS.

Se determinan como variables de estudio, las acciones de sustentabilidad que realiza la empresa que favorecen a nueve de los diecisiete ODS (Naciones Unidas, 2015), los cuales se clasifican en dos categorías: personas y medio ambiente. En la categoría de personas se encuentran las variables de empleo digno (ED); salud, seguridad y bienestar (SSB); igualdad entre géneros (IG); educación inclusiva, equitativa y de calidad (EIEC) y relaciones cordiales entre empresa y pobladores (REP). En la categoría de medio ambiente se encuentran las variables de producción sostenible (PS); acciones para combatir el cambio climático (ACC); restablecer el ecosistema (RE) e infraestructura resiliente (IR). Finalmente, se consideran los valores compartidos como factores determinantes de la confianza en las empresas (Fields, 2002) (Tabla 1). 
Tabla 1. Clasificación de las variables. Fuente propia.

\begin{tabular}{|c|c|c|}
\hline Personas & Medio ambiente & Confianza \\
\hline $\begin{array}{l}\text { 1. Empleo digno (ED) } \\
\text { 2. Salud, seguridad bienestar } \\
\text { (SSB) } \\
\text { 3. Igualdad entre géneros (IG) } \\
\text { 4. Educación inclusiva, } \\
\text { equitativa y de calidad } \\
\text { (EIEC) } \\
\text { 5. Relaciones cordiales entre } \\
\text { empresas y pobladores } \\
\text { (REP) }\end{array}$ & $\begin{array}{l}\text { 6. Producción } \\
\text { sostenible (PS) } \\
\text { 7. Acciones para } \\
\text { combatir el cambio } \\
\text { climático (ACC) } \\
\text { 8. Restablecer el } \\
\text { ecosistema (RE) } \\
\text { 9. Infraestructura } \\
\text { resiliente (IR) }\end{array}$ & $\begin{array}{l}\text { 10. Valores } \\
\text { compartidos (C) }\end{array}$ \\
\hline
\end{tabular}

Para obtener la percepción de la población, se utiliza una encuesta formada por 10 ítems con preguntas cerradas. Las respuestas están formadas con cinco opciones de acuerdo a la escala de Likert. La escala comprende los siguientes valores: 1 a la respuesta "totalmente en desacuerdo", 2 "en desacuerdo", 3 "ni de acuerdo ni en desacuerdo", 4 "de acuerdo" y 5 "totalmente de acuerdo". La estructura del instrumento presenta un segmento con los datos censales y otro con las preguntas. Se utiliza el muestreo aleatorio para aplicar el instrumento.

Para identificar el nivel de contribución de las acciones de la empresa a los ODS, se toma como base la media aritmética obtenida en cada variable. Los valores mayores o iguales a 3.5 serán considerado como una contribución "significativa" al logro de los ODS y los valores menores a 3.5 como una contribución "no significativa". Se define como "significativo" a aquello que refiere importancia sobre algo, por representar o tener valor para alguien. (Real Academia Española, 2001).

La herramienta utilizada para el estudio son los modelos de normalización, para analizar las variables en su propia naturaleza, y chicuadrada para comprender la relación de dependencia entre la confianza (variable de respuesta) y los ODS (variable Explicativa).

\section{Resultados}

La aplicación del instrumento fue en forma aleatoria y de manera directa por los investigadores del trabajo, obteniendo 135 respuestas. 


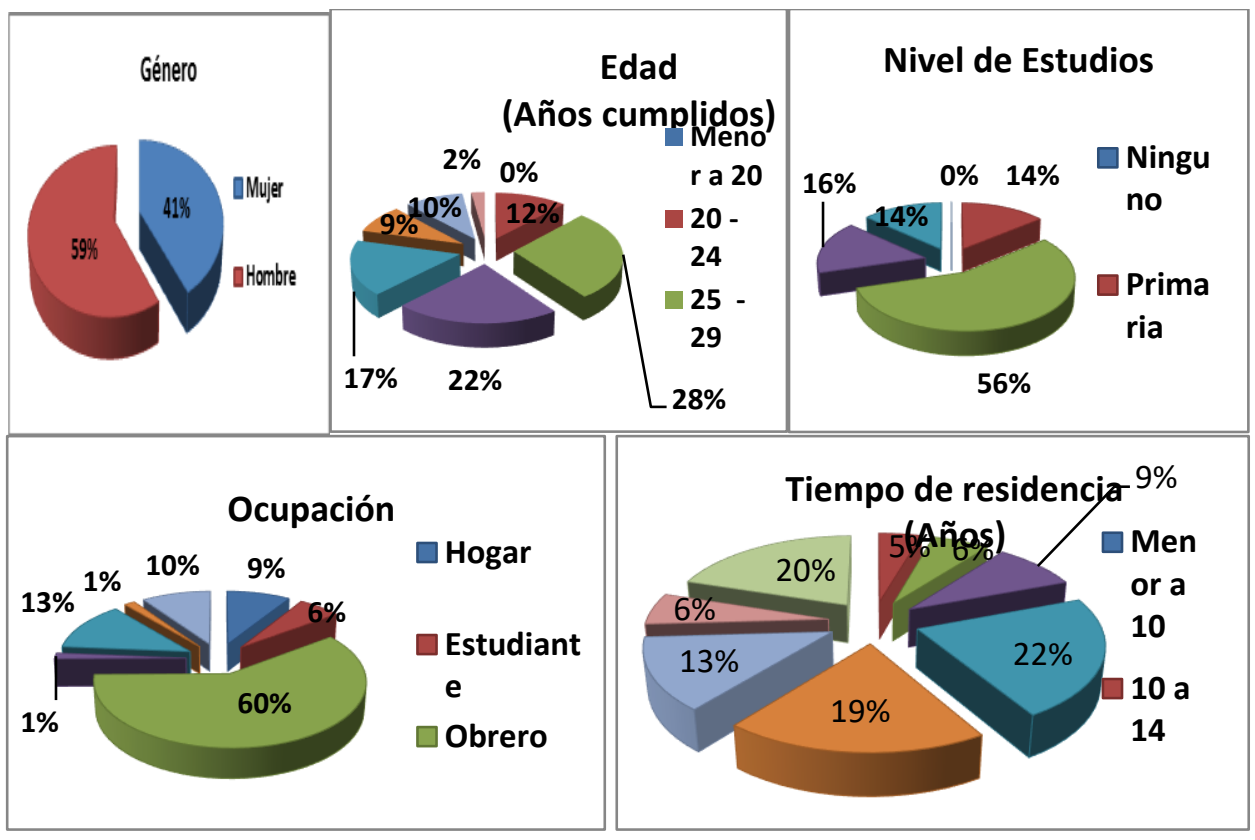

Figura 1. Características de la población. Fuente propia.

Las características más relevantes de la población encuestada (Figura 1) son las siguientes:

- El 59\% son hombres (todos jefes de familias).

- Ninguna persona es menor de 20 años y la mayor parte de la población (67\%) tiene entre 25 y 39 años de edad.

- El $100 \%$ de la población cuenta con formación académica, de los cuales el 70\% el nivel es de educación básica.

- El 60\% de esta población son obreros.

- El total de la población tiene más de 10 años de residir en la localidad donde vive actualmente.

- No existe relación laboral, o de otro tipo de subordinación, entre los encuestados y la empresa.

Los valores obtenidos en la media aritmética por cada variable, así como en los valores compartidos (factores que determinan la confianza), se presentan en la Tabla 2.

De acuerdo a los resultados obtenidos, las acciones en la categoría de "personas" que muestran una contribución significativa al logro de los ODS, son de SSB con un valor de 3.65. Las acciones que presentan una contribución no significativa a los ODS, por haber obtenido valores inferiores a 3.5 son: ED (3.44), IG (2.97), EIEC (3.19) y REP (3.18).

Con relación a los valores obtenidos en la media aritmética, las acciones en la categoría de "medio ambiente" que muestran una contribución significativa al logro de los ODS, son: ACC (3.75), RE (3.90) e IR (3.69). 
Las acciones que presentan una contribución no significativa a los ODS, por haber obtenido un valor inferior a 3.5 son de PS (2.56).

Tabla 2. Media aritmética de las variables. Fuente propia.

\begin{tabular}{|cc|}
\hline Personas & 3.44 \\
Empleo digno (ED) & 3.65 \\
Salud, seguridad bienestar (SSB) & 2.97 \\
Igualdad entre géneros (IG) & 3.19 \\
Educación inclusiva, equitativa y de calidad (EIEC) & 3.18 \\
Relaciones cordiales entre empresas y pobladores (REP) & \\
$\quad$ Medio ambiente & 2.56 \\
Producción sostenible (PS) & 3.75 \\
Acciones para combatir el cambio climático (ACC) & 3.90 \\
Restablecer el ecosistema (RE) & 3.69 \\
Infraestructura resiliente (IR) & \\
Confianza & 3.48 \\
\hline
\end{tabular}

Finalmente, las acciones que realiza la empresa para mostrar valores compartidos con la población, como factor determinante de la confianza en la organización, presentan una contribución no significativa a los ODS, por haber obtenido un valor de 3.48 .

El comportamiento de las variables, de ambas categorías, se muestran a través de las curvas de normalización, donde se analiza su propia naturaleza y se compara su curtosis con la confianza observando lo siguiente:

En la Gráfica 1 se puede apreciar que la variable de SSB es platicúrtica debido a que su valor de curtosis es menor a -0.4 (-0.57). Lo anterior indica que, a pesar de haber sido valorada como significativa; es decir, que las acciones de salud, seguridad y bienestar sí contribuyen al logro de los ODS, el criterio de la población encuestada es altamente disperso. De forma contraria, la variable IG es leptocúrtica debido a que su valor de curtosis es mayor a $0.4(0.53)$. Lo anterior indica que, además de haber sido valorada como no significativa; es decir, que en las actividades que ofrece la empresa a la población no existe un trato de igualdad entre géneros y por tanto no contribuyen al logro de los ODS, el criterio de la población encuestada es altamente unificado. 


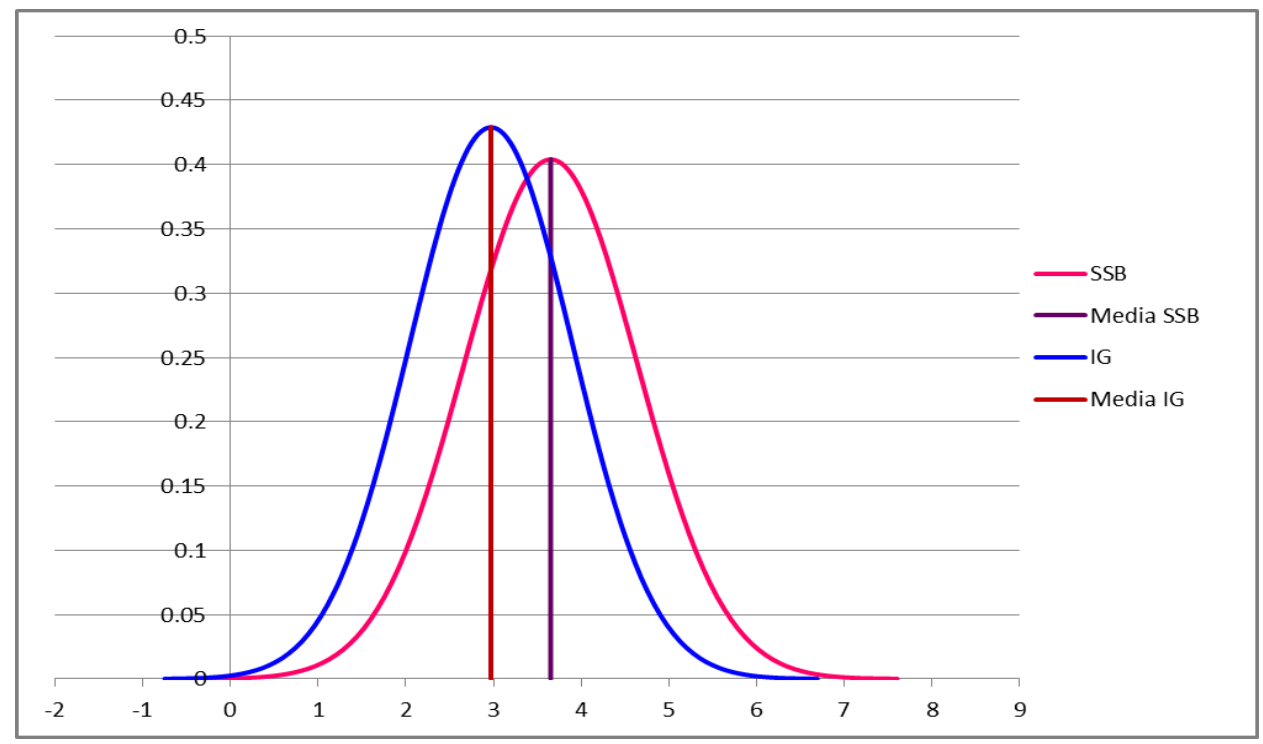

Grafica 1. Normalización de variables SSB e IG. Fuente propia.

La Gráfica 2 muestra la normalización de las variables ED, EIEC y REP con una dispersión normal debido a que sus valores de curtosis son de 0.37, -0.08 y 0.33 , respectivamente. Lo anterior implica que el criterio de la población es imparcial, a pesar de que la variable REP tiende a un criterio más unificado de la población. Sin embargo, todas las acciones que representan estas variables no contribuyen a los ODS por ser no significativas.

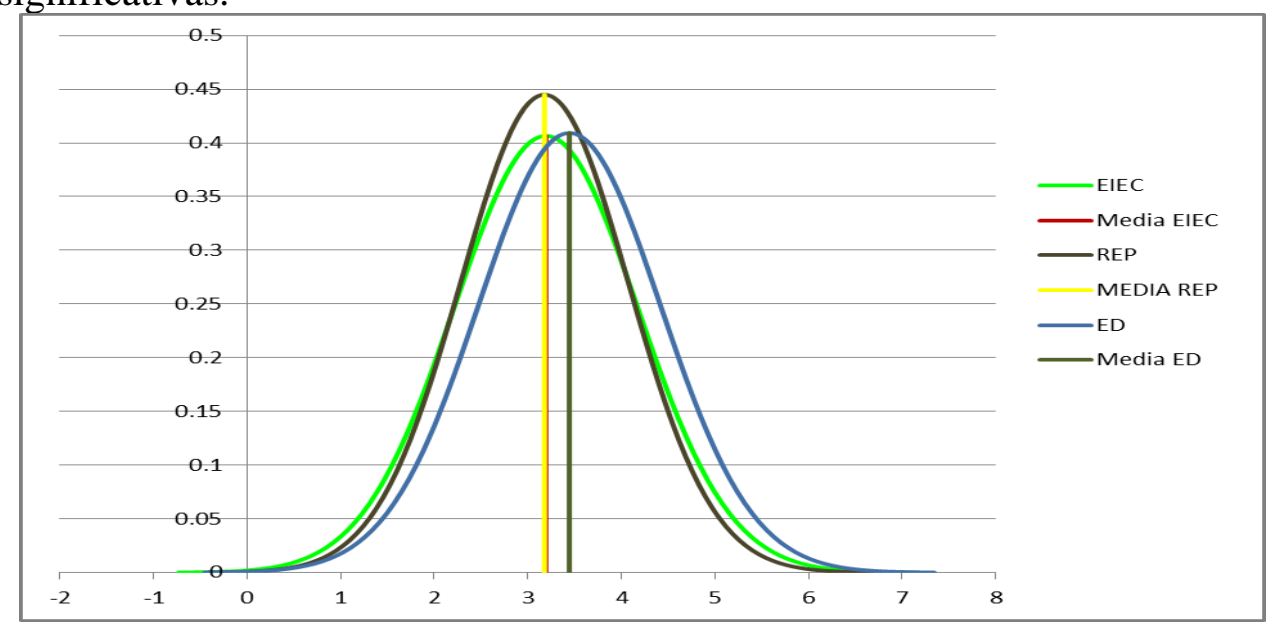

Grafica 2. Normalización de variables EIEC, REP y ED. Fuente propia.

Las acciones para combatir el cambio climático (ACC), restablecer el ecosistema (RE); así como el apoyo que otorga la empresa para la construcción de infraestructuras resilientes (IR); sí contribuyen al logro de 
los ODS, de acuerdo a lo mencionado en los valores de la media aritmética. Sin embargo, de acuerdo a su valor de curtosis (-0.55) en la variable ACC, su distribución es platicúrtica, lo que define que el criterio de la población encuestada es altamente disperso. Sin embargo, las variables RE, IR y PS presentan una distribución normal debido a que sus valores de curtosis son $0.12,-0.18$ y -0.23 , respectivamente; a pesar de que las acciones para realizar una producción sostenible (PS) no contribuyen a los ODS (Grafica 3).

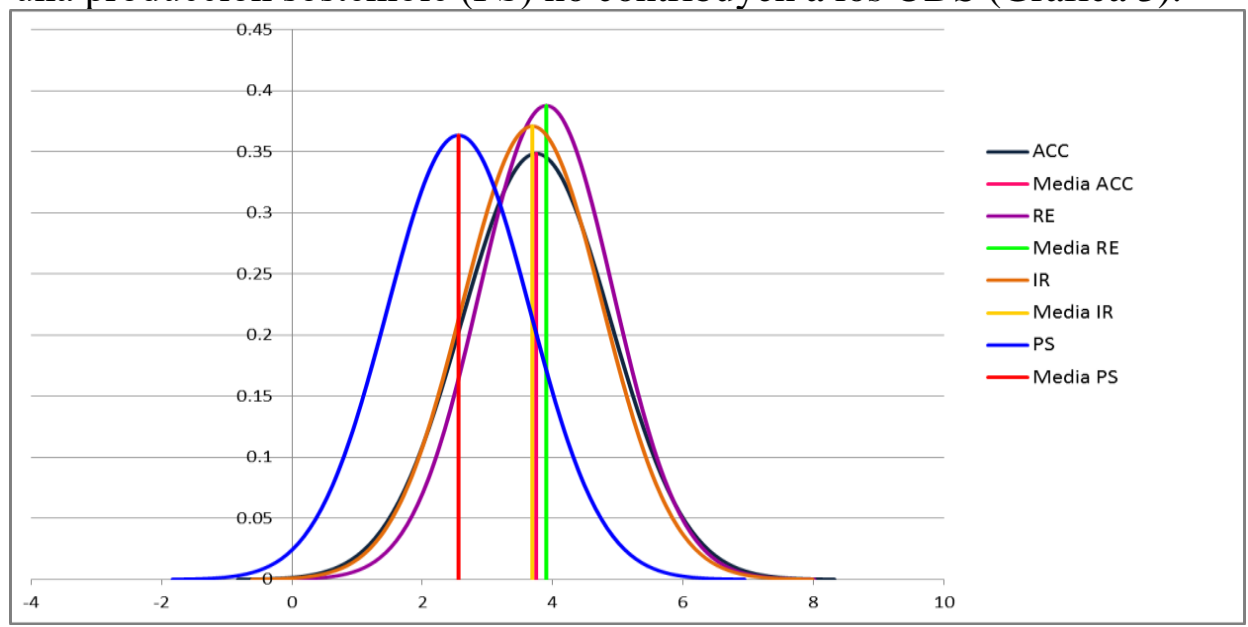

Grafica 3. Normalización de variables ACC, RE, IR y PS. Fuente propia.

Finalmente en la Grafica 4, las acciones que realiza la empresa para mostrar que comparten los mismos valores con los pobladores de las localidades, como factor determinante de la confianza, son percibidas por la población como no significativas y por tanto, no contribuyen a los ODS. Este criterio es imparcial (mesocúrtuca), de acuerdo al valor a su valor de curtosis $(-0.01)$.

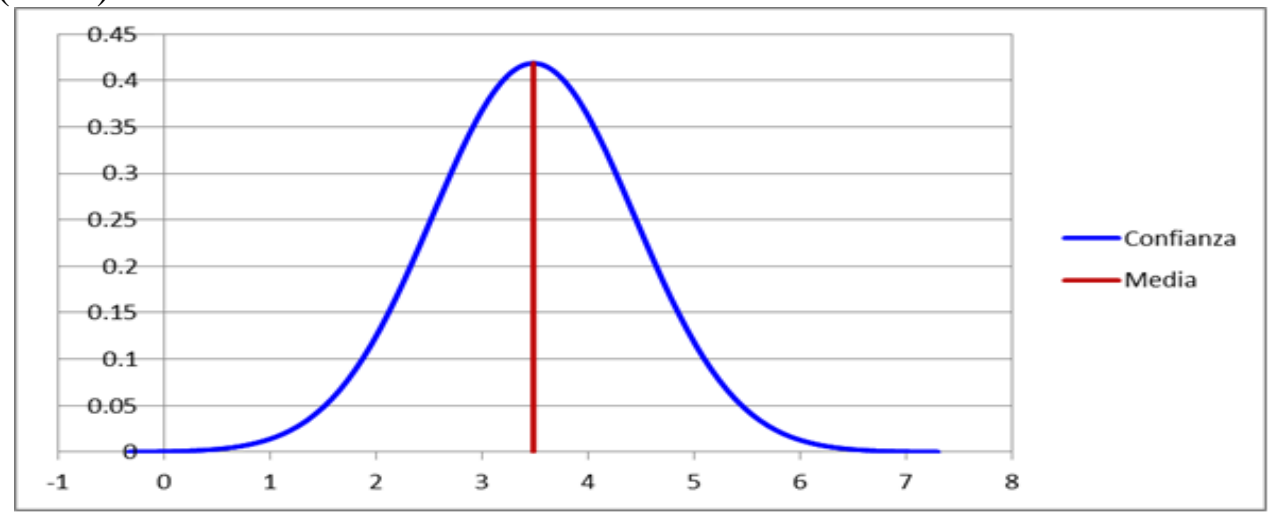

Grafica 4. Normalización de la variable Confianza (C). Fuente propia. 
Por otra parte, al comparar la relación de cada una de las variables de las dos categorías (personas y medio ambiente) con la confianza, a través de la distribución de chi-cuadrado o Pearson, se obtuvieron los resultados que se muestran en la Tabla 3.

Tabla 3. Valores de chi-cuadrado u observado. Fuente propia.

\begin{tabular}{|cc|}
\hline Categorías y subcategorías / variables & Valores \\
Personas & \\
Empleo digno (ED) & 1.8318 \\
Salud, seguridad bienestar (SSB) & 0.7311 \\
Igualdad entre géneros (IG) & 0.6056 \\
Educación inclusiva, equitativa y de calidad (EIEC) & 3.5664 \\
Relaciones cordiales entre empresas y pobladores & 2.8310 \\
(REP) & \\
Medio ambiente & 2.8068 \\
Producción sostenible (PS) & 0.0320 \\
Acciones para combatir el cambio climático (ACC) & 2.5271 \\
Restablecer el ecosistema (RE) & 0.2950 \\
Infraestructura resiliente (IR) & \\
Valor de Chi-teórico $=\mathbf{3 . 8 4 1 4}$ & \\
\hline
\end{tabular}

Debido a que el valor de chi-cuadrado u observado en cada una de las variables asociadas es inferior al valor de chi-teórico; se determina que no existe relación estadística entre éstas y la confianza. Es decir, la confianza de las personas de la población hacia la empresa, no depende de las acciones que ésta realiza para favorecer a las personas o al medio ambiente.

\section{Conclusion}

De acuerdo con el Programa de Naciones Unidas, "los ODS, son un llamado universal a la adopción de medidas para poner fin a la pobreza, proteger el planeta y garantizar que todas las personas gocen de paz y prosperidad. Apoyar la puesta en marcha de la Agenda 2030 es una prioridad importante para el PNUD", afirma la Administradora del PNUD, Helen Clark" (PNU, 2017).

La contribución de las empresas para para el logro de los ODS es fundamental porque constituyen una fuente de crecimiento y desarrollo económico, empleo e innovación. A su vez, al colaborar al logro de los ODS, las empresas obtienen beneficios debido a que su éxito solo puede darse en sociedades estables y prósperas; por lo que las soluciones innovadoras y sostenibles enfocadas en sectores de bajos ingresos, representa una gran oportunidad de negocios para las empresas y un alto beneficio para la sociedad (WBCSD, 2016).

Según el informe de PWC (2015), antes de entrar en vigor los ODS $\left(1^{\circ}\right.$ de enero de 2016), el $92 \%$ de los negocios eran ya conscientes de los ODS, el $72 \%$ de los negocios estaban comenzando a ser conscientes y 
planeaban tomar acciones frente al tema, el 29\% de las empresas ya estaban estableciendo sus metas alineadas a los ODS y el 13\% de los negocios ya habían identificado las herramientas que necesitarían para incluir, alinear y aplicar los ODS a su gestión.

En el mismo sentido, Garcidueñas (2015) señaló a 20 empresas que ya trabajaban para sumarse al logro de los ODS, entre ellas Cemex. A su vez, Cemex, en su reporte de sustentabilidad, informa de manera formal las acciones que realiza para contribuir con los ODS (Cemex, 2016).

No obstante, la incorporación de los ODS en la estrategia empresarial y las acciones que se llevan a cabo para contribuir a su logro, deben promover: confianza entre todas las partes, transparencia en las relaciones, integridad y marcos éticos.

Por tanto, esta investigación tuvo como propósito identificar, si las acciones que realiza la empresa Cemex han contribuido al logro de los ODS en la localidad de Atotonilco de Tula, estado de Hidalgo; y si estas acciones generan confianza en los pobladores de sus comunidades locales.

De acuerdo a las hipótesis planteadas, podemos afirmar que:

La hipótesis de investigación 1 se rechaza. Las acciones que realiza la empresa Cemex, dirigidas al beneficio de las personas no contribuyen al logro de los ODS y las acciones dirigidas a la conservación del medio ambiente sí.

Los resultados obtenidos de las medias aritméticas en cuatro variables comprendidas en la categoría de "personas", fueron no significativas. Lo cual indica que la mayoría de las acciones que la empresa dirige para el beneficio de las personas de la comunidad no contribuyen al logro de los ODS en lo referente a empleo digno (ED); educación inclusiva, equitativa y de calidad (EIEC); así como las relaciones cordiales entre empresa y pobladores (REP). De la misma forma, fueron valoradas como no significativas las actividades que ofrece la empresa a la población con relación a la igualdad entre géneros (IG); por tanto, no contribuyen al logro de los ODS. El criterio manifestado fue altamente unificado de acuerdo a su valor de curtosis (0.53). De manera opuesta, las acciones que favorecen la salud, seguridad y bienestar de las personas (SSB) si resultaron significativas en su contribución a los ODS. Sin embargo, el valor obtenido de curtosis (0.57) muestra que el criterio de la población es altamente disperso.

Por otra parte, los resultados obtenidos de las medias aritméticas en tres variables comprendidas en la categoría de "medio ambiente", si fueron significativas. Por tanto, las acciones que la empresa dirige en beneficio al medio ambiente sí contribuyen al logro de los ODS para restablecer el ecosistema (RE), proporcionar infraestructura resiliente (IR) y combatir el cambio climático (ACC); a pesar de que en éstas últimas, el criterio de la población fue altamente disperso de acuerdo a su valor de curtosis (-0.55). 
Sin embargo, las personas perciben que la producción no es sostenible (PS); debido a que obtuvo un resultado no significativo; y por tanto, no contribuye al logro de los ODS.

La hipótesis de investigación 2 se rechaza. La confianza de las personas de la población hacia la empresa, no depende de las acciones que ésta realiza para contribuir al logro de los ODS.

Los valores observados en todas las variables, a través de la distribución de chi-cuadrado o Pearson, fueron inferiores al valor teórico de chi-cuadrada. Lo anterior muestra que no existe una relación directa entre la confianza y los ODS para la empresa Cemex, en su Planta Atotonilco.

En resumen, los pobladores pueden reconocer que algunas de las acciones que la empresa realiza en favor de las personas o del medio ambiente contribuyan al logro de los ODS; sin embargo, estas acciones no están generando confianza de la población hacia la empresa.

$\mathrm{Si}$ bien, "las empresas que alinean sus prioridades con los ODS, pueden fortalecer su compromiso con los clientes, empleados y con otras partes interesadas; [y] aquellas que no lo hagan, estarán expuestas a crecientes riesgos legales y de reputación" (PMNU, WBCSD y GRI, 2015, p.4). Por tanto, las empresas deben establecer un compromiso auténtico realizando acciones significativas; es decir, congruentes a lo que reportan en sus informes de sustentabilidad y generar un impacto positivo en sus grupos de interés.

Si la empresa Cemex pretende lograr una buena reputación para generar actitudes y comportamientos favorables en las comunidades locales, como uno de los grupos de interés que son clave para su crecimiento y supervivencia, ésta habrá de procurar mayor atención a las acciones que realiza para contribuir al logro de los ODS.

\section{References:}

1. Barney, J. \& Hansen, M. (1994). Trustworthiness as a Source of Competitive Advantage. Strategic Management, 15, 175-190.

2. Barrios, V. (2010, Abril 21). Sostenibilidad económica y social como prioridad para la sustentabilidad ambiental. Recuperado de https://www.gestiopolis.com/sostenibilidad-economica-socialprioridad-sustentabilidad-ambiental/

3. Barroso, F.G. (2008). La responsabilidad social empresarial: un estudio en cuarenta empresas de la ciudad de Mérida, Yucatán. Revista Contaduría y Administración. 226, 73-91.

4. Bourdieu, P. (2000). Las formas del Capital: Capital económico, capital cultural, y capital social. Poder, derecho y clases sociales. Bilbao: Desclee de Brouwer. 
5. Bouten, L., Everaert, P., Van Liedekerke, L., De Moor, L., \& Christiaens, J. (2011). Corporate Social Responsibility Reporting: a Comprehensive Picture?. Accounting Forum, 35(3), 187-204.

6. Cámara Nacional del Cemento (CANACEM) (2017). México. Recuperado de http://canacem.org.mx/plantas/

7. Cementos Mexicanos (Cemex) (2016). Soluciones en Concreto para un Futuro Sostenible. Informe de Desarrollo Sostenible 2015. México. http://www.cemex.com/ES/DesarrolloSustentable/files/InformeDesar rolloSustentableCemex2015.pdf

8. Consejo Empresarial Mundial para el Desarrollo Sostenible (WBCSD). (2016). Contribuyendo a los Objetivos de Desarrollo Sostenible: El enfoque de Negocios Inclusivos. Recuperado de www.wbcsd.org/contentwbc/download/1812/22723

9. Corporate Excellence-Centre for Reputation Leadership (2011). Área Asuntos Públicos. Documento de partida sobre asuntos públicos. ¿Cómo entendemos en Corporate Excellence-Centre for Reputation Leadership los 'Asuntos Públicos' y por qué ahora constituyen una clara oportunidad? (documento interno). Recuperado de DJSI Global Report. http://www.sustainability-index.com/

10. Edelman (2013). Anual Global Study México. Edelman Trust Barometer Recuperado de http://es.slideshare.net/EdelmanMexico/2013-edelmantrustbarometermexico?qid=8753ed79-90b0-47a6-b723$\mathrm{d} 1647 \mathrm{~b} 4 \mathrm{e} 61 \mathrm{c} 0 \& \mathrm{v}=\mathrm{qf} 1 \& \mathrm{~b}=\&$ from_search $=1$

11. Edelman (2014). Global Results. Edelman Trust Barometer Recuperado de http://es.slideshare.net/EdelmanInsights/2014edelman-trust-barometer

12. Edelman (2015). Global Results. Edelman Trust Barometer Recuperado de http://es.slideshare.net/EdelmanInsights/2015edelman-trust-barometer-global-results

13. Edwards, P. (2016). Summary: Global Cement Directory. Report 2017. Global Cement Magazine. 18-28.

14. Fields, D. (2002). Taking the measure of work. A guide to validated scales for organizational trust. Strategic Change, 14, $77-92$.

15. Fombrun, Ch. \& Van, R. (2004). Fame and Fortune: How Successful Companies Build Winning Reputation,. New York: Pearson-Financial Times.

16. Font, X., Walmsley, A., Cogotti, S., McCombes, L., \& Hausler, N. (2012). Corporate social responsibility: The disclosure-performance gap. Tourism Management, 33(6), 1544-1553. 
17. García，J. S. (2012). ¿Legitimidad de la responsabilidad social empresarial? Coloquio Ética y Capitalismo: ¿Responsabilidad Social? Departamento de Administración. Universidad Autónoma Metropolitana. México.

18. Garcidueñas, P. (2015). 20 ejemplos de empresas que trabajan por los objetivos de desarrollo sostenible. Expock. Comunicación de Sustentabilidad y RSE. Recuperado de http://www.expoknews.com/20-ejemplos-de-empresas-que-trabajanpor-los-objetivos-de-desarrollo-sostenible/

19. Hewett, K. \& Bearden, W.O. (2001). Dependence, trust, and relational behavior on the parí of foreign subsidiary marketing operations: Implications for managing global marketing operations. Journal of Marketing, 65(4), 51-66.

20. Instituto Panamericano de Alta Dirección de Empresas (IPADE) \& Gestión Social y Cooperación (GESOC) (2011). Índice de Transparencia en Sustentabilidad Corporativa. Recuperado de http://www.ipade.mx/Documents/cegi/indice-de-transparencia-ensustentabilidad-corporativa-web.pdf

21. Jones, G. R. \& George, J. M. (1998). La experiencia y la evolución de la confianza: implicaciones para la cooperación y el trabajo en equipo. Academy of Management, 23(3), 531-546.

22. Lewicki, R., McAllister, D., \& Bies, R. (1988). Trust and distrust; new relationships and realities. Academy of Management Reivew, 23(3), $438-458$.

23. Martínez, A. (2014). Estudio del sector cementero a nivel mundial y nacional, con particularización de una empresa cementera situada en la Comunidad Valenciana. [Tesis]. Universidad Politécnica de Valencia. Noviembre, Programa de Adminstración y Dirección de Empresas.

24. Montañez, M. \& Gutiérrez, O. (2014). Las prácticas responsables desde la teoría los grupos de interés en las pequeñas y medianas empresas. Ra Ximhai, Revista de Sociedad, Cultura y Desarrollo Sustentable, 10(3), 27-38.

25. Naciones Unidas (1987). Informe de la Comisión Mundial sobre el Medio Ambiente y el Desarrollo. Nuestro futuro común. Recuperado de

http://www.exteriores.gob.es/Portal/es/PoliticaExteriorCooperacion/ Desarrollosostenible/Documents/Informe\%20Brundtland\%20(En\%20 ingl\%C3\%A9s).pdf

26. Naciones Unidas (2015). Asamblea General. Resolución A/70/L.1. Documento final de la cumbre de las Naciones Unidas para la aprobación de la agenda para el desarrollo después de 2015. 
Transformar nuestro mundo: la Agenda 2030 para el Desarrollo Sostenible. Recuperado de http://www.un.org/ga/search/view_doc.asp?symbol=A/70/L.1\&Lang $=\mathrm{S}$

27. Pacto Mundial de las Naciones Unidas (PMNU), Consejo Empresarial Mundial para el Desarrollo Sostenible (WBCSD) \& Global Reporting Initiative (GRI) (2015). SDG Compass: La guía para la acción empresarial en los ODS. Recuperado de http://www.pactomundial.org/sdm_downloads/sdg-compass-la-guiala-accion-empresarial-los-ods/

28. Price Waterhousecoopers (PWC) (2015). Make it your Business: Engaging with the Sustainable Development Goals. Recuperado de: https://www.pwc.com/gx/en/sustainability/SDG/SDG\%20Research_ FINAL.pdf

29. Programa de Naciones Unidas para el Desarrollo (PNU) (2017). Pbjetivos de Desarrollo Sostenible. Recuperado de http://www.undp.org/content/undp/es/home/sustainabledevelopment-goals.html

30. Quevedo, E. (2003). Reputación y Creación de Valor. Una Relación Circular. $1^{\circ}$ edición. Madrid: Paraninfo.

31. Remacha, M. (2017). Empresa y objetivos de desarrollo sostenible. Cátedra CaixaBank de Responsabilidad Social Corporativa. 34, 128.

32. Rojas, L. M. D. \& Marin S.P. (2006). Aproximaciones a la Medición de Confianza. DYNA: Revista de la Facultad de Minas. Universidad Nacional de Colombia. Sede Medellín, 73(150), 119-130.

33. Saldaña, R. A. (2010). Responsabilidad social empresarial: Hacia una agenda de investigación en México. Administración $y$ organizaciones, 12(24), 75-89. 